\title{
Theoretical study of quasiparticle inelastic lifetimes as applied to aluminum
}

\author{
I. A. Nechaev, ${ }^{1, *}$ I. Yu. Sklyadneva,,${ }^{1,2}$ V. M. Silkin, ${ }^{3}$ P. M. Echenique, ${ }^{1,3}$ and E. V. Chulkov ${ }^{1,3}$ \\ ${ }^{1}$ Donostia International Physics Center (DIPC), P. de Manuel Lardizabal, 4, 20018, San Sebastián, Basque Country, Spain \\ ${ }^{2}$ Institute of Strength Physics and Materials Science, Prospekt Academicheski 2/1, 634021, Tomsk, Russia \\ ${ }^{3}$ Departamento de Física de Materiales, Facultad de Ciencias Químicas, UPV/EHU and Centro Mixto CSIC-UPV/EHU, Apartado 1072, \\ 20080 San Sebastián, Basque Country, Spain
}

(Received 11 March 2008; revised manuscript received 8 July 2008; published 11 August 2008)

\begin{abstract}
We present a comparative analysis of different microscopic approaches to quasiparticle properties in metals. Aluminum is chosen as an application object, since it exhibits characteristics many of which are well-described in the jellium model. Within this model, we consider how different levels of physical elaboration of the electron-electron interaction affect the imaginary part of the quasiparticle self-energy and the quasiparticle renormalization constant. Also, we present ab initio calculations of the quasiparticle lifetime in crystalline aluminum with the use of both the linear muffin-tin orbital method and the plane-wave pseudopotential theory. To complete the picture of inelastic scattering effects in aluminum, we report first-principles calculations on electron-phonon interaction and on the phonon mediated contribution to the lifetime. The total inelastic lifetime broadening is compared with experimental data known from the literature.
\end{abstract}

DOI: 10.1103/PhysRevB.78.085113

PACS number(s): 71.10.-w, 63.20.kd

\section{INTRODUCTION}

Quasiparticle excitations in metals play an important role in a rich variety of physical and chemical phenomena. ${ }^{1}$ As an example, one can mention energy transfer in photochemical reactions, desorption and oxidation of molecules at surfaces, spin transport within bulk metals, across interfaces, and at surfaces. One of the key properties of quasiparticle excitations is their lifetime (inverse quasiparticle decay rate) which characterizes the duration of these excitations. Along with the quasiparticle velocity, the lifetime determines such measure of influence of the excitation as the mean free path.

The decay rate $\Gamma_{\text {tot }}$ of a quasiparticle can be presented as a sum of the following contributions:

$$
\Gamma_{\text {tot }}=\Gamma_{\mathrm{e}-\mathrm{e}}+\Gamma_{\mathrm{e}-\mathrm{ph}} .
$$

Here, the first term, $\Gamma_{\mathrm{e}-\mathrm{e}}$, is the contribution from the inelastic electron-electron (e-e) scattering mechanism that includes several decay channels concerned with charge-density, spindensity, singlet-pair, and triplet-pair fluctuations. ${ }^{2-5}$ The second term, $\Gamma_{\mathrm{e}-\mathrm{ph}}$, represents the contribution from inelastic electron-phonon (e-ph) scattering. This contribution is usually considered as comparable with the electron-electron part only close to the Fermi level, within a typical phonon energy $\hbar \omega_{D}\left(\omega_{D}\right.$ is the Debye cutoff energy). ${ }^{6,7}$ However, $\Gamma_{\text {e-ph }}$ can play an important role at higher electron energies for metals with large e-ph coupling. ${ }^{8}$ Moreover, unlike $\Gamma_{\mathrm{e}-\mathrm{e}}$ which experiences very weak temperature dependence, the e-ph contribution significantly increases with temperature and can even exceed the e-e part. ${ }^{8}$

At present, there are many theoretical approaches to the quasiparticle decay rate which differ one from another by the level of physical elaboration of the electron-phonon and electron-electron interactions. For example, similar to the electron-phonon interaction, the electron-electron one can be considered as realized through the exchanging by a number of varieties of "bosons" (the aforementioned fluctuations) composed of electrons and holes, which themselves strongly interact through the exchange by other bosons. ${ }^{3}$ Depending on how many varieties of these bosons are involved in the description of the e-e interaction within an approach, one obtains different results for contribution of the exchangecorrelation effects to quasiparticle properties.

Basically, the mentioned difference can be studied within an application of the approaches to a given system. A comparison of the obtained results will allow one to reveal factors, which influence mainly quasiparticle properties upon moving from one approach to another. However, the majority of the approaches to $\Gamma_{\mathrm{e}-\mathrm{e}}$ can be effectively applied to a prototype homogeneous system only. In applying them to real metal systems, one encounters difficulties in taking properly into consideration real band-structure effects. This means that, apart from construction of a model of the e-e interaction, real materials give an additional challenge. As a result, a consistent comparison of the approaches applied to the same material is an important matter.

In this paper, we give a comparative analysis of a wide spectrum of approaches to $\Gamma_{\mathrm{e}-\mathrm{e}}$ as applied to aluminum. The choice of such a material is motivated by the fact that aluminum possesses the free-electron-like band structure, and therefore many properties of this simple metal can be described well within the jellium model. In support of the latter, one can mention, e.g., the Fermi energy as determined by $\mathrm{x}$-ray photoemission, ${ }^{9}$ the plasmon dispersion and its very small anisotropy, ${ }^{10,11}$ and the stopping power for protons and antiprotons. ${ }^{12}$ As a consequence, aluminum can be considered as a prototype material that allows one to test a theoretical approach to quasiparticle dynamics of jellium.

The approaches considered in this work are chosen to reflect the present state of the art of quasiparticle dynamics of metals. In order to make the paper self-sustained, we give a brief description of the approaches existing in the literature, starting from the well-known $G_{0} W_{0}$ approximation, passing through different methods taking into account the contribution of both charge- and spin-density fluctuations, and ending up with the so-called generalized $G_{0} W_{0}$ approximation. Within the description, we outline both the approxi- 
mations, which consider the mentioned contributions by means of the spin-symmetric and spin-antisymmetric localfield (LF) factors, and the approaches, which are based on a variational method and do not involve quantities defined outside their scope. As a development of one of such approaches, we propose an approximation that consistently includes multiple electron-hole scattering effects both in the screening properties and in the decay rate.

As regards the phonon-induced lifetime broadening, we evaluate $\Gamma_{\mathrm{e}-\mathrm{ph}}$ expressed in terms of the Eliashberg spectral function in the quasielastic approximation at different temperature. Apart from the fact that $\Gamma_{\text {e-ph }}$ is of interest as a contribution to the quasiparticle decay rate, this lifetime broadening can play an important role in definition of the proper approach to $\Gamma_{\mathrm{e}-\mathrm{e}}$ by comparing to available experimental data.

The paper is organized as follows: In Sec. II, we briefly describe all the considered approaches to the e-e decay rate within many-body perturbation theory. Within the jellium model, we compare them as applied to aluminum. In this section, we also present results of our first-principles calculations for the e-e quasiparticle lifetime in $\mathrm{Al}$ which take into account both charge- and spin-density fluctuations. Section III contains our $a b$ initio results on the e-ph decay rate evaluated at different temperatures. In Sec. IV, we compare the obtained total decay rate with experimental relaxation-time data existing in the literature. The conclusions are also given in Sec. IV. Unless stated otherwise, atomic units are used throughout, i.e., $e^{2}=\hbar=m=1$.

\section{ELECTRON-ELECTRON SCATTERING}

Within many-body theory of condensed matter, a study of quasiparticle properties, including the electron-electron lifetime $\tau(\omega)=\Gamma_{\mathrm{e}-\mathrm{e}}^{-1}(\omega)$ as a function of exciting energy $\omega$, usually rests on the solution of the well-known closed set of Hedin's coupled integral equations. ${ }^{13}$ These equations (see, e.g., Ref. 14) relate the Green's function of a system under the study

$$
G(1,2)=G_{0}(1,2)+G_{0}(1,3) \Sigma(3,4) G(4,2)
$$

to the quasiparticle self-energy

$$
\Sigma(1,2)=i G(1,3) W(1,4) \Lambda(3,2,4)
$$

and, through the latter, to the screened interaction

$$
W(1,2)=v_{c}(1,2)+v_{c}(1,3) P(3,4) W(4,2),
$$

which is determined by the irreducible polarizability (with the factor of 2 for spin)

$$
P(1,2)=-2 i G(1,3) G(4,1) \Lambda(3,4,2),
$$

where the vertex function

$$
\begin{aligned}
\Lambda(1,2,3)= & \delta(1-2) \delta(2-3) \\
& +\frac{\delta \Sigma(1,2)}{\delta G(4,5)} G(4,6) G(7,5) \Lambda(6,7,3) .
\end{aligned}
$$

Here, considering the paramagnetic state, the standard short-

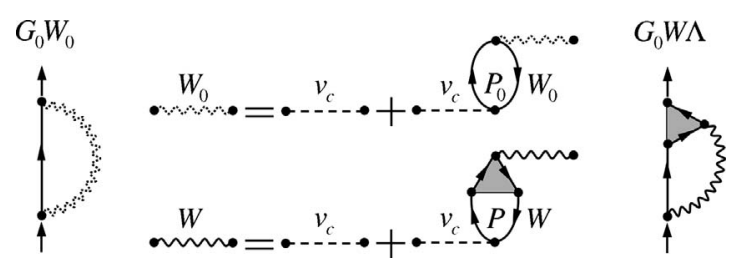

FIG. 1. A diagrammatic representation of the self-energy and the screened interaction treated in the $G_{0} W_{0}$ and $G_{0} W \Lambda$ approximations. The solid lines with arrows represent the zeroth-order Green's function $G_{0}$. The dotted and solid wiggly lines signify the RPA screened interaction $W_{0}$ and the screened interaction with the inclusion of vertex corrections, respectively. The shaded triangles indicate the vertex $\Lambda$ evaluated in the local approximation.

hand notation $1=\left(\mathbf{r}_{1}, t_{1}\right)$ for space-time coordinates is used. Repeated variables are integrated, unless they appear on both sides of the equations. In Eq. (2), the Green's function $G_{0}$ of zeroth order is built on a complete set of single-particle states at the Hartree (mean-field) level. The interaction (4) describes a dynamic spin-independent dielectric screening of the bare Coulomb interaction $v_{c}$ between two test charges "nonidentical" to electrons in the Fermi sea.

According to Hedin's concept, by solving these equations iteratively, one generates the perturbation diagrammatic expansion for the self-energy, vertex function, and irreducible polarizability in terms of the screened interaction.

\section{A. Jellium model}

\section{1. $G_{0} W_{0}$ approximation}

Starting from $\Sigma=0$ and, consequently, $\Lambda(1,2,3)$ $=\delta(1-2) \delta(2-3)$, the first cycle of the iterative solution of Hedin's Eqs. (2)-(6) ends up by modeling the quasiparticle self-energy as $\Sigma(1,2)=i G_{0}(1,2) W_{0}(1,2)$ with the screened interaction $W_{0}$ obtained within the random-phase approximation (RPA), where the irreducible polarizability is presented by $P_{0}(1,2)=-2 i G_{0}(1,2) G_{0}(2,1)$. Such an expression for the self-energy constitutes the so-called $G_{0} W_{0}$ approximation.

Taking into consideration homogeneity of jellium, in momentum space the mentioned self-energy and irreducible polarizability can be rewritten as

$$
\Sigma(k)=\frac{i}{(2 \pi)^{4}} \int d q G_{0}(k-q) W_{0}(q),
$$

and

$$
P_{0}(q)=-\frac{2 i}{(2 \pi)^{4}} \int d p G_{0}(p-q) G_{0}(p),
$$

respectively. Here and in the following, the four-momentum variable $q$ as a shorthand for $(\mathbf{q}, \omega)$ is used. The RPA screened interaction is given by

$$
W_{0}(q)=v_{c}(|\mathbf{q}|)+v_{c}(|\mathbf{q}|) P_{0}(q) W_{0}(q) \text {. }
$$

Feynman diagrams of the considered quantities are shown in Fig. 1. 
TABLE I. Values of the parameters used in the analytical expressions [Eqs. (12)-(14)] for the spinsymmetric and spin-antisymmetric local-field factors for $r_{s}=2.07$ corresponding to the case of aluminum.

\begin{tabular}{rcccccccc}
\hline \hline$Q_{\uparrow \uparrow}$ & $Q_{\uparrow \downarrow}$ & $A$ & $B$ & $C$ & $\alpha_{c}$ & $\beta$ & $A_{1}$ & $g_{0}$ \\
\hline 1.5014 & 3.2741 & 0.2679 & 0.8320 & 0.0491 & 0.1059 & 0.3794 & 0.1739 & 0.1385 \\
\hline \hline
\end{tabular}

\section{Local-field corrections}

Within the RPA at the Hartree level, we deal with the screening properties of the system of independent electrons which do not feel any effect of exchange and correlation. However, the existence of a local exchange-correlation (XC) hole around electrons in the Fermi sea leads to the reduction in the interaction between electrons, and, consequently, to changes in the system response. Therefore, to get a more realistic screening picture, within the paradigm of linear response and locality of XC corrections, the many-body localfield factors $\mathcal{G}_{\uparrow \uparrow}(q)$ and $\mathcal{G}_{\uparrow \downarrow}(q)$ are introduced. ${ }^{15}$ The factor $\mathcal{G}_{\uparrow \uparrow}(q)$ mainly accounts for the Pauli principle effect, while $\mathcal{G}_{\uparrow \downarrow}(q)$ is responsible for taking into account the Coulomb correlation between antiparallel spin electrons. As a rule, these factors are tabulated and parametrized by using quantum Monte Carlo (QMC) calculations for the homogeneous electron gas.

For paramagnetic systems, the symmetric and antisymmetric combinations, $\mathcal{G}_{ \pm}(q)=\left[\mathcal{G}_{\uparrow \uparrow}(q) \pm \mathcal{G}_{\uparrow \downarrow}(q)\right] / 2$, of the many-body local-field factors are introduced. The spinsymmetric $\mathcal{G}_{+}$determines the density (charge-charge) response function ${ }^{5,15}$

$$
R_{d}(q)=\frac{P_{0}(q)}{1-v_{c}(|\mathbf{q}|)\left[1-\mathcal{G}_{+}(q)\right] P_{0}(q)}
$$

whereas $\mathcal{G}_{-}$enters the definition of the magnetic (longitudinal spin-spin) response function ${ }^{5,15}$

$$
R_{S_{z} S_{z}}(q)=\frac{P_{0}(q)}{1+v_{c}(|\mathbf{q}|) \mathcal{G}_{-}(q) P_{0}(q)} .
$$

A simple way to analytically express $q$ dependence of the local-field factors is to cast them into energy-independent Hubbard-type forms. In this case, the aforementioned combinations can be represented as ${ }^{16}$

$$
\mathcal{G}_{ \pm}^{\mathrm{IP}}(q)=\frac{1}{2}\left[\frac{|\mathbf{Q}|^{2}}{|\mathbf{Q}|^{2}+Q_{\uparrow \uparrow}^{2}} \pm \frac{|\mathbf{Q}|^{2}}{|\mathbf{Q}|^{2}+Q_{\uparrow \downarrow}^{2}}\right]
$$

Here and in the following we use the dimensionless momentum $\mathbf{Q}=\mathbf{q} / k_{F}$, where the Fermi wave vector $k_{F}=\left(\alpha r_{s}\right)^{-1}$ is determined by the electron density parameter $r_{s}$ given by $r_{s}^{3}=3 /(4 \pi n)\left[n\right.$ being the electron density and $\left.\alpha=(4 / 9 \pi)^{1 / 3}\right]$. The parameters $Q_{\uparrow \uparrow}$ and $Q_{\uparrow \downarrow}$ (see Table I) are such that the compressibility and susceptibility sum rules ${ }^{5}$ are satisfied by $\mathcal{G}_{+}^{\mathrm{IP}}(q)$ and $\mathcal{G}_{-}^{\mathrm{IP}}(q)$ at the small- $q$ limit, respectively.

In Ref. 17, the following more complex analytical expression for the spin-symmetric local-field factor has been proposed:

$$
\mathcal{G}_{+}^{\mathrm{CDOP}}(Q)=C|\mathbf{Q}|^{2}+\frac{B|\mathbf{Q}|^{2}}{g+|\mathbf{Q}|^{2}}+\alpha_{c}|\mathbf{Q}|^{4} e^{-\beta|\mathbf{Q}|^{2}},
$$

where the Hubbard-type term [with $g=B /(A-C)$ ] is present nevertheless. The parameters of such a representation of $\mathcal{G}_{+}$ are determined in such a way as to meet the requirements of the correct asymptotic behaviors for both small- and large- $q$ limits and to reproduce QMC results at intermediate $q$ values. These parameters evaluated with the use of the correlation energy of Ref. 18 are listed in Table I.

In formal analogy with Eq. (13), the spin-antisymmetric local-field factor that also behaves correctly at the small- and large- $q$ limits can be approximated as ${ }^{5,19}$

$$
\mathcal{G}_{-}^{\mathrm{SG}}(Q)=C|\mathbf{Q}|^{2}+\frac{B_{1}|\mathbf{Q}|^{2}}{g_{1}+|\mathbf{Q}|^{2}},
$$

where $g_{1}=B_{1} /\left(A_{1}-C\right)$ and $B_{1}=B-1+2 g_{0}$ with the pair correlation function at zero-interelectronic distance $g_{0}$ parameterized, e.g., by the form of Ref. 20 (see Table I).

In Fig. 2, we show the mentioned spin-symmetric and

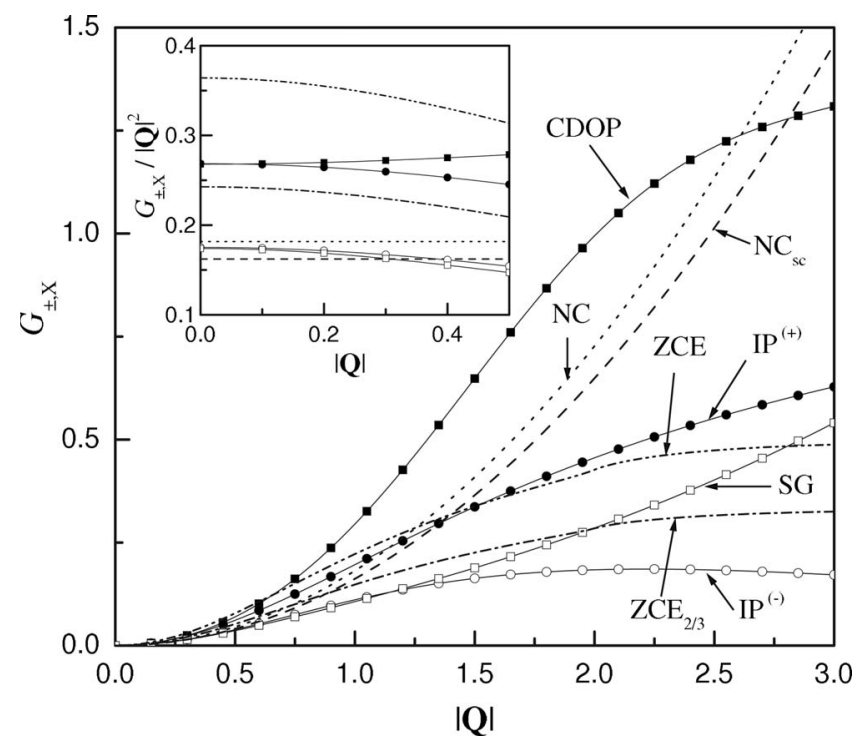

FIG. 2. The local-field factors $\mathcal{G}_{+}, \mathcal{G}_{-}$, and $\mathcal{G}_{X}$ as functions of momentum $|\mathbf{Q}|$ at $r_{s}=2.07$. Here and in the following the notation CDOP signifies $\mathcal{G}_{+}$taken in the analytic form of Ref. 17 [Eq. (13)]. The spin-antisymmetric local-field factor taken from Ref. 19 [Eq. (14)] is marked as SG. $\mathrm{IP}^{(+)}$and $\mathrm{IP}^{(-)}$denote $\mathcal{G}_{+}$and $\mathcal{G}_{-}$of Ref. 16 [Eq. (12)], respectively. The long-wave limit form for $\mathcal{G}_{X}$ obtained in Ref. 21 is marked by NC (its self-consistent variant is denoted by the additional subscript sc). The notation ZCE signifies $\mathcal{G}_{X}$ corresponding to the approach of Ref. 22 (the subscript $2 / 3$ points out its modification done in Ref. 21). Inset: all of these local-field corrections divided by squared momentum $|\mathbf{Q}|$. 
spin-antisymmetric local-field factors as functions of $|\mathbf{Q}|$. As is seen from the figure, the spin-symmetric "CDOP" and "IP(+)" factors (as well as the spin-antisymmetric "SG" and "IP( ${ }^{(-)}$" ones) are rather different in their dependencies on $|\mathbf{Q}|$. Nevertheless, due to the correct small- $q$ asymptotic behavior, both analytic expressions for $\mathcal{G}_{+}$(or for $\mathcal{G}_{-}$) provide similar local-field corrections at small values of $|\mathbf{Q}|$ (see inset of Fig. $2)$. Other curves presented in the figure we discuss below.

\section{3. $G_{0} W$ approximation}

The Fourier transform of the irreducible polarizability [Eq. (5)] that determines screening properties of the system leads to $^{23}$

$$
P(q)=-\frac{2 i}{(2 \pi)^{4}} \int d p G(p-q) G(p) \Lambda(p, q) .
$$

The RPA irreducible polarizability [Eq. (8)] corresponds to the lowest order of the vertex function. This means that taking the exchange and correlation effects into consideration of $P$ implies evaluating the vertex function in higher orders of the perturbation theory than it occurs in the RPA. One way to evaluate these so-called vertex corrections to $P_{0}$ is to formally reduce them to the spin-symmetric local-field factor. $^{24,25}$ As was shown in Ref. 26, it can be done, e.g., by using a variational approach within a local approximation (see also Refs. 27-29). In such a way, one sums a set of infinite classes of diagrams that leads to the vertex function dependent on one four-momentum only, i.e.,

$$
\Lambda(q)=\left[1+v_{c}(|\mathbf{q}|) \mathcal{G}_{+}(q) P_{0}(q)\right]^{-1} .
$$

This local form for $\Lambda$ essentially simplifies calculations of $P$. Actually, with the use of the zeroth-order Green's function, it allows one to rewrite Eq. (15) as

$$
P(q)=P_{0}(q) \Lambda(q)
$$

that, in turn, provides the density response function $R_{d}(q)$ $=P(q)+P(q) v_{c}(|\mathbf{q}|) R_{d}(q)$ in the form of Eq. (10).

The Fourier-transformed dynamically screened interaction (see Fig. 1),

$$
W(q)=v_{c}(|\mathbf{q}|)+v_{c}(|\mathbf{q}|) P(q) W(q),
$$

is determined by the irreducible polarizability [Eq. (17)] that contains the vertex corrections [Eq. (16)] enhancing the density response. This interaction differs from that in the RPA and, when used in the $G_{0} W_{0}$ formula in place of $W_{0}$, allows one to go beyond the $G_{0} W_{0}$ approximation. Such an approximation is denoted as the $G_{0} W$ one.

\section{4. $G_{0} W \Lambda$ approximation}

Starting from the Fourier transform of Eq. (3),

$$
\Sigma(k)=\frac{i}{(2 \pi)^{4}} \int d q G(k-q) W(q) \Lambda(k, q),
$$

it is natural to suppose that at the next level of complexity the same vertex corrections should be included both in the screened interaction and in the self-energy. ${ }^{27}$ In this case,
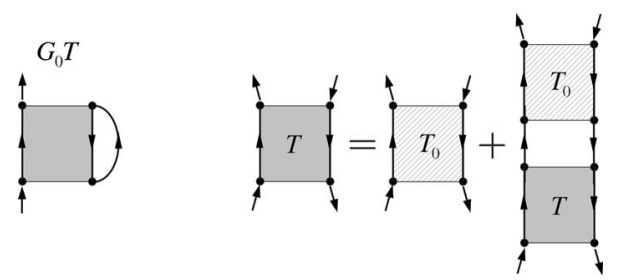

FIG. 3. Feynman diagrams of the self-energy treated as an integral over the e-h scattering amplitude $T$ that is defined by the BetheSalpeter equation with the irreducible e-h vertex $T_{0}$. As before, solid lines with arrows represent the zeroth-order Green's function $G_{0}$.

using the zeroth-order Green's function, the screened interaction [Eq. (18)] with $P$ of Eq. (17), and the vertex function [Eq. (16)], we arrive at the self-energy of the $G_{0} W \Lambda$ approximation (Feynman diagrams are shown in Fig. 1). The latter exploits the same $G_{0} W_{0}$ formula, where the resulting screened interaction $W_{\text {e-t }}(q)=W(q) \Lambda(q)$ is used instead of $W_{0}$. This $W_{\mathrm{e}-\mathrm{t}}$ is interpreted as an electron-test charge interaction ${ }^{15}$ in contrast to the test charge-test charge one, $W$.

On rewriting the electron-test charge interaction as $W_{\mathrm{e}-\mathrm{t}}(q)=v_{c}(|\mathbf{q}|)+v_{c}^{2}(|\mathbf{q}|)\left[1-\mathcal{G}_{+}(q)\right] R_{d}(q)$, it becomes clear that, as well as in the $G_{0} W_{0}$ and $G_{0} W$ approximations, quasiparticle properties are determined solely by the density response. In other words, the self-energy includes only the contribution of charge fluctuations. This means that a different expression is needed for the self-energy in order to take additionally into account the contribution of spin-density fluctuations.

\section{5. $G_{0} W_{0} \& T$ approximation}

Owing to the correspondence between electron-hole multiple-scattering events and spin fluctuations (see, e.g., Refs. 30-33), their contribution can be included in the selfenergy by treating the latter as an integral over the four-point e-h scattering amplitude ${ }^{2,3}$ shown in Fig. 3. A simple way to calculate the self-energy in such a manner within a study that rests on Hedin's concept is to express the scattering amplitude $T$ in terms of the dynamically screened interaction as a sort of $T_{0}$ [a ladder approximation, where $T_{0}(1,2 \mid 3,4)$ $=W(1,2) \delta(1-3) \delta(2-4)]$. At that, to preserve all the advantages of the $G_{0} W_{0}$ approximation, the integral over $T$ can be considered as an additional term to the $G_{0} W_{0}$ self-energy. ${ }^{22,34}$ In this case, in order to avoid double counting, as in Ref. 3, the scattering amplitude should start from the third order in the screened interaction. ${ }^{21,34}$

Such an additional term can be regarded as vertex corrections to the $G_{0} W_{0}$ self-energy. As in the $G_{0} W \Lambda$ case, a local approximation can be developed to solve the Bethe-Salpeter equation that describes the e-h scattering amplitude. In Ref. 34 , it has been done by using a variational approach yielding the amplitude $T$ which in momentum space and in the case of a uniform system depends only on the four-momentum transfer along the electron-hole channel. As a result, the additional self-energy term [hereafter referred to as the $T$-matrix contribution and denoted by $\left.\Sigma^{T}\right]$ has the $G_{0} W_{0}$ formula (7) with $W_{0}$ replaced by the scattering amplitude 


$$
T(q)=\widetilde{W}(q) P_{0}(q) \widetilde{W}(q)\left[\frac{1}{1+\frac{1}{2} P_{0}(q) \widetilde{W}(q)}-1\right],
$$

where the factor of 2 for spin is implied. The local interaction

$$
\widetilde{W}(q)=-2\left[P_{0}(q)\right]^{-1} P_{1}(q)\left[P_{0}(q)\right]^{-1}
$$

is related to the first-order exchange diagram in the irreducible polarizability diagrammatic expansion

$$
P_{1}(q)=-2 \int d k d p \kappa_{q}(k) W(k-p) \kappa_{q}(p),
$$

where $\kappa_{q}(p)=i G_{0}(p-q) G_{0}(p) /(2 \pi)^{4}$. Consequently, this interaction can be identified with the many-body local-field factor $\mathcal{G}_{X}=\widetilde{W} / 2 v_{c}$ evaluated within the first-order perturbation theory (see, e.g., Ref. 35), when the spin-symmetric and spin-antisymmetric local-field factors are equal.

At the small- $q$ limit, the local interaction becomes $q$-independent and the factor $\mathcal{G}_{X}$ shown in Fig. 2 as the "NC" curve demonstrates the quadratic behavior, i.e., $\mathcal{G}_{X}=A_{X}|\mathbf{Q}|^{2}$, where the constant $A_{X}=W k_{F}^{2} /(8 \pi)$. With the use of the RPA screened interaction $W_{0}$ in Eq. (22), at $r_{s}=2.07$ the constant $A_{X}$ is equal to 0.1817 .

Within the presented $G_{0} W_{0} \& T$ approach, by replacing the local interaction $\widetilde{W}(q)$ by the static screened interaction $W_{0}(\mathbf{q}, 0)$, we can reproduce an approximation made in Ref. 22. This approximation is based on a first-principles study ${ }^{36,37}$ of the strength $U$ of Hubbard's contact interaction, where different matrix elements of the static screened interaction are analyzed. With such a local interaction, the factor $\mathcal{G}_{X}$ is equal to $W_{0}(\mathbf{q}, 0)\left[2 v_{c}(|\mathbf{q}|)\right]^{-1}$ (see the "ZCE" curve in Fig. 2). At the small- $q$ limit, it leads to $\mathcal{G}_{X}=A_{X}|\mathbf{Q}|^{2}$ with $A_{X}$ $=0.3641$ that, as was shown in Ref. 21, overestimates the ladder diagrams contribution at $r_{s}=2.07$.

\section{6. $G_{0} W \Lambda \& T$ ladder approximation}

Within the previous $G_{0} W_{0} \& T$ approach, which takes into account some vertex corrections to the $G_{0} W_{0}$ self-energy, the screening properties of the system are described within the RPA, whereas in, e.g., the $G_{0} W \Lambda$ approximation, one gets a more realistic screening picture. In order to eliminate this defect, we go beyond the RPA. As a development of the $G_{0} W_{0} \& T$ approach, here we propose an approximation that allows one to take into account more ladder diagrams along the line of Hedin's expansion of both the self-energy and the irreducible polarizability.

First, note that the vertex function of Eq. (6) with the first-order functional derivation $\delta \Sigma(1,2) / \delta G(4,5)$ $=W(1,2) \delta(1-4) \delta(2-5)$ can be expressed in terms of the e-h scattering amplitude of the ladder approximation. As a result, in momentum space, with the use of the aforementioned variational solution to the Bethe-Salpeter equation for the amplitude $T$ and with the zeroth-order Green's function, we arrive at the vertex function $\Lambda_{X}(q)=[1$ $\left.+v_{c}(|\mathbf{q}|) \mathcal{G}_{X}(q) P_{0}(q)\right]^{-1}$, where $\mathcal{G}_{X}=\widetilde{W} / 2 v_{c}$. Through Eqs. (17) and (18) the screened interaction $W$ in Eq. (22) can include local-field corrections with the aid of the vertex function

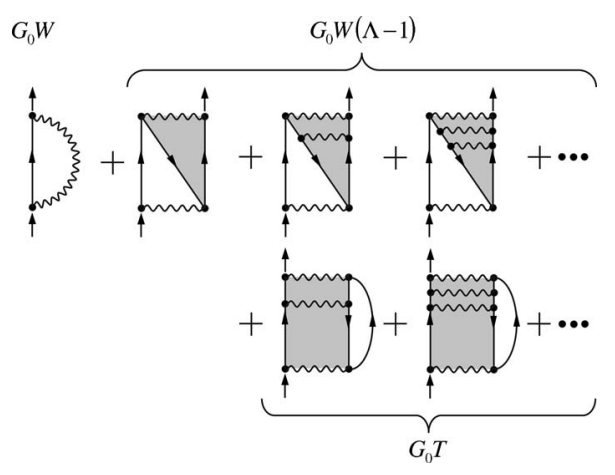

FIG. 4. Feynman diagrams of the Hedin self-energy diagrammatic expansion taken into consideration within the $G_{0} W \Lambda \& T$ ladder approximation. The wiggly lines signify the dynamically screened Coulomb interaction $W$. The solid lines with arrows represent the zeroth-order Green's function $G_{0}$. The shaded areas indicate the vertex $\Lambda$ and the scattering amplitude $T$ evaluated in the local approximation.

$\Lambda_{X}(q)$. This means that one can reach a self-consistency between $\mathcal{G}_{X}$ and $W$ which, on the one hand, contains local-field corrections provided by $\mathcal{G}_{X}$ and, on the other hand, determines this local-field factor through $\widetilde{W}$. In this case the constant $A_{X: s c}=0.1622$ (sc denotes self-consistent) becomes slightly smaller than that found with $W_{0}$ (see Fig. 2 and note the proximity of the "NC" and " $\mathrm{NC}_{\mathrm{sc}}$ " factors $\mathcal{G}_{X}$ to $\mathcal{G}_{-}$at small values of $|\mathbf{Q}|$ ).

Second, with the use of such a self-consistent local-field factor the leading term of the self-energy can be evaluated within the $G_{0} W \Lambda$ approximation with $\Lambda_{X}(q)$. At that, the additional term $\Sigma^{T}$ should also be expressed in terms of the sc factor $\mathcal{G}_{X}$. The resulting self-energy represents a sum of an infinite number of ladder diagrams shown in Fig. 4. Thus, within such an approximation, one can include consistently multiple electron-hole scattering effects both in the screened interaction and in the self-energy.

In the case of the approximation of Ref. 22 the coefficient $A_{X: \mathrm{sc}}=\frac{2}{3} A_{X}$, which allows one to reasonably estimate the considered diagrams contribution in the entire metallic density range. ${ }^{21}$ The local-field factor $\mathcal{G}_{X: \mathrm{sc}}=W_{0}(\mathbf{q}, 0)\left[3 v_{c}(|\mathbf{q}|)\right]^{-1}$ is shown in Fig. 2 as the " $\mathrm{ZCE}_{2 / 3}$ " curve. As is seen from the inset of the figure, at small momentum, such a factor $\mathcal{G}_{X: s c}$ is quite close to $\mathcal{G}_{+}^{\mathrm{CDOP}}$. Thus, the "ZCE $\mathrm{Z}_{2 / 3}$ " factor provides a way to include real band-structure effects, when one models local-field corrections in $a b$ initio calculations of screening properties. ${ }^{38}$

\section{Generalized $G_{0} W_{0}$ approximation}

A study of the self-energy as the integral over the e-h scattering amplitude shown in Fig. 3 can also rest on an expansion in terms of the bare Coulomb interaction (see, e.g., Ref. 3). In this case, the integral can be considered as vertex corrections to the Hartree-Fock self-energy. A specific example is the approach proposed in Ref. 2. In this approach called "mother-of-all $G W$ approximations" in Ref. 25 or "generalized $G W$ approximation" in Ref. 5, there are three types of the irreducible e-h vertex. Each of them determines 
the corresponding scattering e-h amplitude. Within the local approximation these amplitudes read as

$$
\begin{aligned}
& T^{( \pm)}(q)=\frac{T_{0}^{( \pm)}(q)}{1+P_{0}(q) T_{0}^{( \pm)}(q)}, \\
& T^{(t)}(q)=\frac{T_{0}^{(t)}(q)}{1+\frac{1}{2} P_{0}(q) T_{0}^{(t)}(q)} .
\end{aligned}
$$

Thus one can take into consideration the contributions of the charge $(+)$, the longitudinal spin $(-)$, and the transverse spin $(t)$ decay channels. Definition of $T_{0}^{( \pm)}$and $T_{0}^{(t)}$ is based on a suggestion in the spirit of the approach of Ref. 15 that these irreducible vertices can be identified $^{2}$ with the local-field factors through $T_{0}^{(+)}(q)=-v_{c}(|\mathbf{q}|)\left[1-\mathcal{G}_{+}(q)\right]$, $T_{0}^{(-)}(q)=v_{c}(|\mathbf{q}|) \mathcal{G}_{-}(q)$, and $T_{0}^{(t)}(q)=v_{c}(|\mathbf{q}|) \mathcal{G}_{t}(q)$.

Considering the integral over the scattering amplitudes [Eqs. (23) and (24)] as an additional term with respect to the Hartree-Fock self-energy, $T^{( \pm)}$and $T^{(t)}$ should start, at least, from the second order in $v_{c}$ to avoid double counting. As a result, again, owing to the local approximation, we arrive at the $G_{0} W_{0}$ expression (7) for the self-energy, where instead of $W_{0}$ one has the so-called effective electron-electron interaction ${ }^{2,5,15}$

$$
\begin{aligned}
V_{\mathrm{eff}}(q)= & v_{c}(|\mathbf{q}|)+\left\{v_{c}(|\mathbf{q}|)\left[1-\mathcal{G}_{+}(q)\right]\right\}^{2} R_{d}(q) \\
& +\left\{v_{c}(|\mathbf{q}|) \mathcal{G}_{-}(q)\right\}^{2} R_{S_{z} S_{z}}(q)+\left\{v_{c}(|\mathbf{q}|) \mathcal{G}_{t}(q)\right\}^{2} R_{S_{+} S_{-}}(q),
\end{aligned}
$$

with

$$
R_{S_{+} S_{-}}(q)=\frac{\frac{1}{2} P_{0}(q)}{1+v_{c}(|\mathbf{q}|) \mathcal{G}_{t}(q) \frac{1}{2} P_{0}(q)}
$$

being the transverse spin-response function. ${ }^{2}$

To compare this approach with those considered above, we modify the mentioned self-energy by rewriting interaction (25) as $V_{\text {eff }}(q)=W(q) \Lambda^{\prime}(q)+V_{m}(q)$ with $W$ of Eq. (18), $\Lambda^{\prime}=1+[\Lambda-1]\left[2-\mathcal{G}_{+}\right]$, and

$$
V_{m}(q)=3 \frac{v_{c}^{2}(|\mathbf{q}|) \mathcal{G}_{-}^{2}(q) P_{0}(q)}{1+v_{c}(|\mathbf{q}|) \mathcal{G}_{-}(q) P_{0}(q)},
$$

where the equivalence $\mathcal{G}_{t}(q)=2 \mathcal{G}_{-}(q)$ for the paramagnetic state is implied. Thus we split up the self-energy into the contribution of charge fluctuations $\Sigma^{d}$ having the $G_{0} W \Lambda$-like formula and the contribution of spin fluctuations $\Sigma^{m}$ given by the $G_{0} W_{0}$-like expression. Such a splitting allows us to analyze these contributions separately.

The interaction (25) can be also rewritten ${ }^{25}$ as $V_{\text {eff }}(q)$ $=W_{\mathrm{e}-\mathrm{t}}(q) \Lambda(q)+V_{\mathrm{LAD}}(q)$, where $V_{\mathrm{LAD}}(q)=V_{d}(q)+V_{m}(q)$ with

$$
V_{d}(q)=\frac{v_{c}^{2}(|\mathbf{q}|) \mathcal{G}_{+}^{2}(q) P_{0}(q)}{1+v_{c}(|\mathbf{q}|) \mathcal{G}_{+}(q) P_{0}(q)} .
$$

As a result, we reveal here that, within an approach in which the spin-symmetric and spin-antisymmetric local-field factors are equal (for example, $\mathcal{G}_{X}$ as in the first-order perturbation theory), the interaction $V_{\mathrm{LAD}}$ represents $T(q)$ of Eq. (20) starting from the second order and not from the third one.

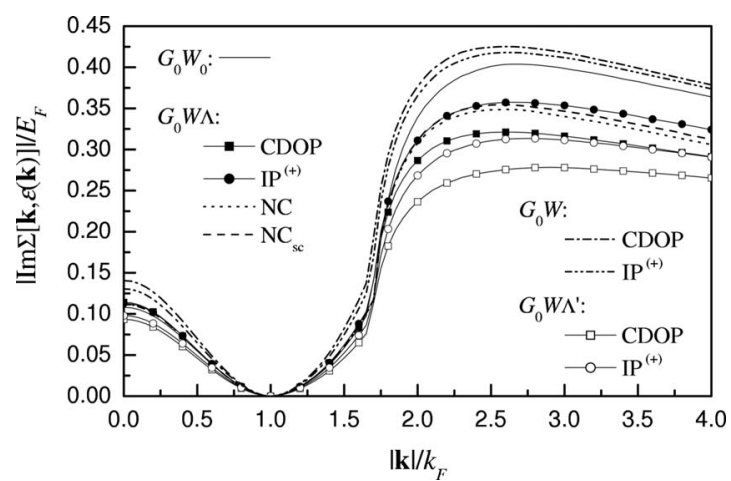

FIG. 5. The absolute value of the imaginary part of the selfenergy (the density channel term) as a function of momentum $|\mathbf{k}| / k_{F}$ evaluated at $\omega=\boldsymbol{\epsilon}(\mathbf{k})$ within the considered approaches.

This means that the scattering amplitude [Eq. (20)] contains three parts coming from the contribution of spin fluctuations and one part-from charge fluctuations. Together with $W_{\mathrm{e}-\mathrm{t}}$, as it used in the $G_{0} W \Lambda \& T$ ladder approximation, such an amplitude gives an interaction similar to that proposed in Ref. 25, where it is considered as a more correct interaction than $V_{\text {eff }}$ containing the term $W_{\text {e-t }} \Lambda=W \Lambda^{2}$.

\section{Numerical results and comparison}

In order to address the question of what factors mainly affect quasiparticle properties upon moving from one approach to another, we have performed extensive calculations of the quasiparticle self-energy and the quasiparticle lifetime, defined as ${ }^{39}$

$$
\tau_{\mathbf{k}}^{-1}=2 Z_{\mathbf{k}}|\operatorname{Im} \Sigma[\mathbf{k}, \epsilon(\mathbf{k})]|,
$$

at $r_{s}=2.07$ that corresponds to the case of aluminum. In Eq. (29), $Z_{\mathbf{k}}$ is the renormalization constant

$$
Z_{\mathbf{k}}=\left[1-\left.\frac{\partial \operatorname{Re} \Sigma(\mathbf{k}, \omega)}{\partial \omega}\right|_{\omega=\epsilon(\mathbf{k})}\right]^{-1},
$$

which determines the spectral weight of the quasiparticle, and $\epsilon(\mathbf{k})=\mathbf{k}^{2} / 2$ being the noninteracting energy. First, we examine the imaginary part of the self-energy at the fourmomentum $[\mathbf{k}, \epsilon(\mathbf{k})]$.

In Fig. 5, we show the contribution of charge-density fluctuations to $\operatorname{Im} \Sigma[\mathbf{k}, \epsilon(\mathbf{k})]$ in units of the Fermi energy $E_{F}$ as a function of momentum. The contribution was calculated without (the $G_{0} W_{0}$ approximation) and with the inclusion of the local-field corrections. As is evident from the figure, compared to the $G_{0} W_{0}$ approximation, the quantity of interest becomes greater within the $G_{0} W$ approximation. It is caused by the fact that the inclusion of the local-field corrections enhances the density response. Within the $G_{0} W \Lambda$ approach, in the region of excitations of single e-h pairs, this enhancement is almost totally reduced by inserting the vertex corrections, i.e., by replacing $W$ by the electron-test charge screened interaction $W_{\mathrm{e}-\mathrm{t}}$ that provides an additional $\mathrm{XC}$ interaction of a test charge with electrons in the Fermi sea. In the energy region, where a quasiparticle can decay into plasmons, this replacement ends up with a decrease in values of 


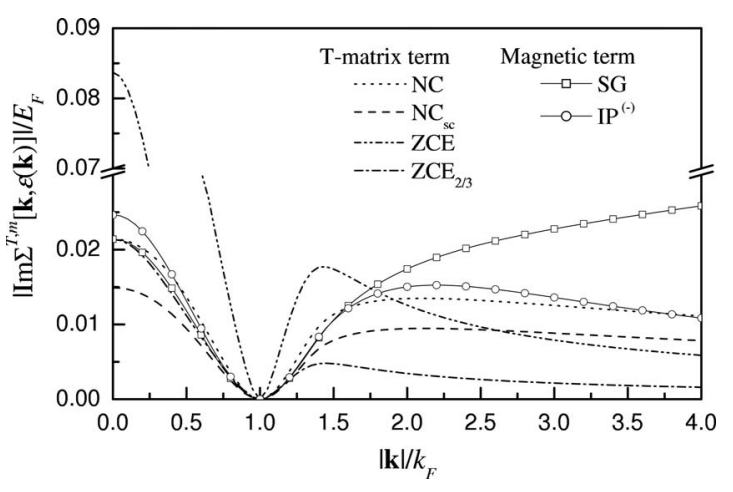

FIG. 6. The absolute value of the imaginary part of the $T$-matrix contribution $\Sigma^{T}$ and the magnetic channel term $\Sigma^{m}$ as a function of momentum $|\mathbf{k}| / k_{F}$ evaluated at $\omega=\boldsymbol{\epsilon}(\mathbf{k})$ with different local-field corrections.

$|\operatorname{Im} \Sigma[\mathbf{k}, \epsilon(\mathbf{k})]|$ even with respect to the $G_{0} W_{0}$ curve. It is caused by narrowing of plasmon peaks in $\omega$-dependence of the imaginary part of the self-energy (see, e.g., Ref. 40). Note that this decrease is rather sensitive to the form taken for the factor $\mathcal{G}_{+}$: Stronger local XC fields (see Fig. 2) cause the $G_{0} W(\Lambda-1)$ term to be larger.

The $G_{0} W \Lambda^{\prime}$ formula yields an even larger decrease in $|\operatorname{Im} \Sigma[\mathbf{k}, \epsilon(\mathbf{k})]|$ both in the single e-h pair excitation region and in the plasmon emission one. It can be explained by the fact that in this case the term additional to the $G_{0} W$ selfenergy is characterized by $\left[\Lambda^{\prime}-1\right]=[\Lambda-1]\left[2-\mathcal{G}_{+}\right]$. This means that in comparison with the $G_{0} W \Lambda$ case the term lowering the $G_{0} W$ curve goes up by a factor of $\left[2-\mathcal{G}_{+}\right]$.

In Fig. 6, we show the imaginary part of $\Sigma^{T}$ and $\Sigma^{m}$. Considering the $T$-matrix contribution first, one can see that this term is quite sensitive ${ }^{21}$ to the form chosen for the factor $\mathcal{G}_{X}$. Actually, small changes in $\mathcal{G}_{X}$, as it occurs when we use the " $\mathrm{NC}_{\mathrm{sc}}$ " factor in place of the "NC" one (see Fig. 2), result in visible decreasing $\left|\operatorname{Im} \Sigma^{T}\right|$ practically at any momentum $|\mathbf{k}|$, except for the vicinity of $k_{F}$. In this vicinity the "NC" and " $\mathrm{NC}_{\mathrm{sc}}$ " results are very close both to each other and to the " $\mathrm{ZCE}_{2 / 3}$ " curve. This is important because in contrast to the local interaction [Eq. (21)] the approximation $\widetilde{W}(q)$ $=2 W(\mathbf{q}, 0) / 3$ makes $a b$ initio calculations of the $T$-matrix contribution feasible. ${ }^{41}$ The use of the local interaction of Ref. 22 (see the "ZCE" curve) yields the $T$-matrix contribution that differs significantly in value and behavior.

As distinct from $\Sigma^{T}$ starting from the third order in $\widetilde{W}$ $=2 v_{c} \mathcal{G}_{X}$, the magnetic term $\Sigma^{m}$ starts from the second order in $v_{c} \mathcal{G}_{-}$[see Eq. (27)]. At that, $\Sigma^{T}$ including the contribution of spin fluctuations and a fraction of the charge channel contribution has the factor of 4 , whereas $\Sigma^{m}$ accounting for the magnetic channel term only is armed with the factor of 3 . Nevertheless, in spite of these differences, the "NC" and "IP(-)" terms are of the same magnitude and demonstrate similar behavior. The deviation of the magnetic term with the different local-field corrections becomes particularly evident only at $|\mathbf{k}|>2.0 k_{F}$, i.e., far in the plasmon emission region.

Now we consider the quasiparticle lifetime [Eq. (29)]. The obtained results are shown in Fig. 7 for several approaches. In the figure, we plot the lifetime as a function of the exciting energy $\left(E-E_{F}\right)$ ranging from zero to $3.5 \mathrm{eV}$ (the

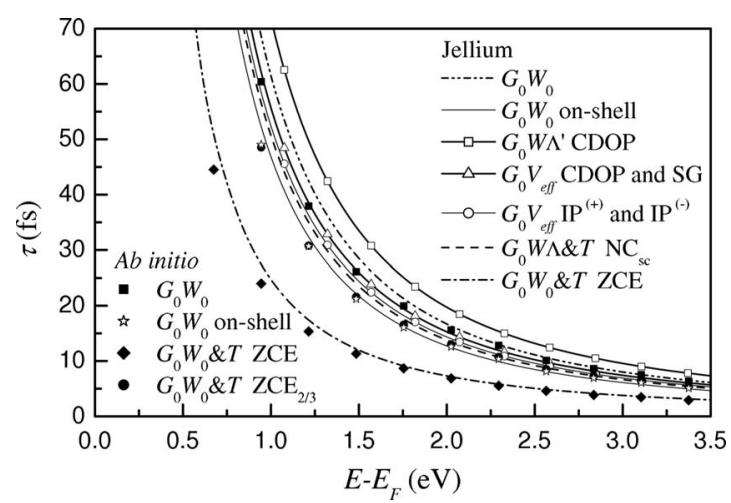

FIG. 7. The electron lifetime $\tau$ as a function of the exciting energy $E-E_{F}$.

range of $|\mathbf{k}| / k_{F}$ from 1.0 to $\left.\sim 1.14\right)$. In order to give a possibility to easily compare and reproduce the presented curves, we take into consideration the fact that, within this excitingenergy range, the scaled lifetime $\tau \times\left(E-E_{F}\right)^{2}$ demonstrates nearly linear behavior in $\left(E-E_{F}\right)$ for all the considered approaches. This means that one can use the linear fit

$$
\tau \times\left(E-E_{F}\right)^{2}=D+S \times\left(E-E_{F}\right)
$$

to reproduce such a behavior. Table II contains our detailed results on the scaled lifetime linear fitting in the mentioned energy range. Here, as a reference mark, one can use the Quinn-Ferrell formula ${ }^{42}$

$$
\tau_{\mathrm{QF}} \times\left(E-E_{F}\right)^{2}=36\left(3 \pi^{2} / 2\right)^{-1 / 3} r_{s}^{-5 / 2}
$$

that gives $D_{\mathrm{OF}}=42.6932 \mathrm{fs} \cdot \mathrm{eV}^{2}$ at $r_{s}=2.07$.

In Table II, the renormalization constant $Z_{\mathbf{k}}$ of Eq. (30) at $k_{F}$ (denoted as $Z_{F}$ ) is also presented. Here, as a reference mark, we can regard the constant $Z_{F}^{\mathrm{GZ}}=0.745$ found for $r_{s}$ $=2.07$ within the parametrization ${ }^{43}$ of the momentum distribution $^{44}$ of the uniform electron gas in its Fermi-liquid regime. In the considered energy range, $Z_{\mathbf{k}}$ varies slightly with the momentum $\mathbf{k}$. This means that, in order to obtain the on-shell scaled lifetime [with $Z_{\mathbf{k}}=1$ in Eq. (29)], one can multiply the presented $D$ and $S$ by the corresponding $Z_{F}$.

As follows from Table II, taking the XC effects into account within the $G_{0} W$ approach results in shortening the lifetime in comparison with that in the $G_{0} W_{0}$ case, in spite of decreasing $Z_{F}$. It is caused by the increase in $|\operatorname{Im} \Sigma|$ (see Fig. 5 ). As regards the $G_{0} W \Lambda$ case, one can see that this approximation depends weakly on the form chosen for the spinsymmetric local-field factor (the ratio $R$ clearly shows that the parameter $D$ changes within $\sim 2 \%$ ) and gives results close to those in the $G_{0} W_{0}$ approximation (see also Refs. 27, 40, and 45). That is the reason why in Fig. 7 we do not show the lifetimes obtained within the $G_{0} W \Lambda$ approximation.

A somewhat different situation is observed in the case of the $G_{0} W_{0} \& T$ approximation. In comparison with the $G_{0} W_{0}$ lifetime, due to the inclusion of the $T$ matrix, $\tau$ becomes noticeably shorter. The maximum lifetime shortening corresponds to the approach of Ref. 22 (see the "ZCE" case in Table II and in Fig. 7). Note that the smallest $Z_{F}$ is obtained also in this case, while the use of the " $\mathrm{ZCE}_{2 / 3}$ " self- 
TABLE II. Parameters $D$ and $S$ of the linear fitting [Eq. (31)] of the scaled lifetime and the renormalization constant $Z_{F}$ at the Fermi wave vector $k_{F}$ evaluated in the considered approaches with the use of different LF corrections. $R$ represents the ratio between $D$ in the $G_{0} W_{0}$ approach and this parameter in the corresponding approach.

\begin{tabular}{|c|c|c|c|c|c|}
\hline Approach & LF corrections & $\begin{array}{c}D \\
\left(\mathrm{fs} \cdot \mathrm{eV}^{2}\right)\end{array}$ & $\begin{array}{c}S \\
(\text { fs } \cdot e V)\end{array}$ & $Z_{F}$ & $R$ \\
\hline \multicolumn{6}{|l|}{$G_{0} W_{0}$} \\
\hline & None & 56.2713 & 5.3819 & 0.76 & 1.00 \\
\hline \multicolumn{6}{|l|}{$G_{0} W$} \\
\hline & CDOP & 47.8690 & 4.0252 & 0.74 & 1.18 \\
\hline & $\mathrm{IP}^{(+)}$ & 50.8109 & 4.4686 & 0.75 & 1.11 \\
\hline \multicolumn{6}{|l|}{$G_{0} W \Lambda$} \\
\hline & CDOP & 57.7073 & 5.7088 & 0.77 & 0.98 \\
\hline & $\mathrm{IP}^{(+)}$ & 56.0002 & 5.3767 & 0.77 & 1.01 \\
\hline & $\mathrm{NC}$ & 56.7122 & 5.5110 & 0.77 & 0.99 \\
\hline & $\mathrm{NC}_{\mathrm{sc}}$ & 56.5789 & 5.4832 & 0.77 & 1.00 \\
\hline \multicolumn{6}{|l|}{$G_{0} W_{0} \& T$} \\
\hline & $\mathrm{ZCE}$ & 19.8410 & 4.8361 & 0.73 & 2.84 \\
\hline & $\mathrm{ZCE}_{2 / 3}$ & 40.8488 & 5.3854 & 0.76 & 1.38 \\
\hline & $\mathrm{NC}$ & 42.1833 & 4.2696 & 0.75 & 1.33 \\
\hline & $\mathrm{NC}_{\mathrm{sc}}$ & 45.8245 & 4.5450 & 0.75 & 1.23 \\
\hline \multicolumn{6}{|l|}{$G_{0} W \Lambda \& T$} \\
\hline & $\mathrm{NC}_{\mathrm{sc}}$ & 46.0464 & 4.6175 & 0.76 & 1.22 \\
\hline \multicolumn{6}{|l|}{$G_{0} W \Lambda^{\prime}$} \\
\hline & CDOP & 64.7112 & 7.1842 & 0.79 & 0.87 \\
\hline & $\mathrm{IP}^{(+)}$ & 60.9492 & 6.3502 & 0.78 & 0.92 \\
\hline \multicolumn{6}{|l|}{$G_{0} V_{\text {eff }}$} \\
\hline & $\mathrm{CDOP}$ and $\mathrm{SG}$ & 50.0421 & 5.6466 & 0.78 & 1.13 \\
\hline & $\mathrm{IP}^{(+)}$and $\mathrm{IP}^{(-)}$ & 47.2844 & 5.1230 & 0.77 & 1.19 \\
\hline
\end{tabular}

consistent local interaction yields the renormalization constant that is the same as in the $G_{0} W_{0}$ approximation.

The main factor which in the considered energy range governs a magnitude of the $T$-matrix contribution and the magnetic term is the long-wave behavior of the local-field factor (see inset of Fig. 2). The point is that after an integration over angles in the formula defining the imaginary part of $\Sigma^{T}$ or $\Sigma^{m}$ the resulting $|\mathbf{q}|$-dependent integrand has a maximum at $\sim 0.25 k_{F}$ and $\sim 0.30 k_{F}$ in the "SG" and "ZCE" case, respectively, or at $\sim 0.5 k_{F}$ for the " $\mathrm{NC}_{\mathrm{sc}}$ " $T$-matrix term. At that, integrating over momentum up to $1.0 k_{F}$, we obtain $\sim 75 \%$ of the "SG" magnetic term, 90\% of the "ZCE" $T$-matrix contribution, and $\sim 50 \%$ of $\left|\operatorname{Im} \Sigma^{T}\right|$ with the use of the " $\mathrm{NC}_{\mathrm{sc}}$ " local-field factor. This means, first, that the shortest lifetime provided by the approach of Ref. 22 (see Fig. 7) is caused by large values of $\widetilde{W}$ at the small- $q$ limit (see inset of Fig. 2, where the "ZCE" curve lies even above the $\mathcal{G}_{+}$ one). Second, in the "NC" and "NC ${ }_{\mathrm{sc}}$ " cases, some overestimation of the ladder diagrams is present, because the small four-momentum transfer limit form for $\mathcal{G}_{X}$ is used.

Note that using the "NC" local-field factor we arrive at the $G_{0} W_{0} \& T$ curve which coincides with the on-shell $G_{0} W_{0}$ one (the parameter $D \approx D_{\mathrm{QF}}$ ). According to the results of Ref. 21 , such a coincidence will occur for $r_{s}$ values ranging from
2 to 56. Taking into account the small real part of the $T$-matrix term, ${ }^{21}$ we can infer that the on-shell $G_{0} W_{0}$ approximation $\left[Z_{\mathbf{k}}=1\right.$ in Eq. (29)] ensures the leading contribution to many-body corrections to the Hartree singleparticle states and implicitly includes decay channels which are not implied by the $G_{0} W_{0}$ diagram.

With the use of the " $\mathrm{NC}_{\text {sc }}$ " local interaction, the $G_{0} W \Lambda \& T$ ladder approach modifies slightly the results obtained in the $G_{0} W_{0} \& T$ case. This modification makes the quasiparticle lifetime a little longer and brings $Z_{F}$ to its $G_{0} W_{0}$ value. As compared to the corresponding $G_{0} W \Lambda$ results, this ladder approach shows how the $T$-matrix contribution expressed in terms of the screened interaction with the inclusion of exchange effects by means of $\mathcal{G}_{X}$ influences the lifetime.

As is evident from Table II and Fig. 7, in the $G_{0} V_{\text {eff }}$ approach the leading $G_{0} W \Lambda^{\prime}$ term, which represents the contribution of charge fluctuations, leads to the lifetime that, in contrast to all the cases analyzed before, noticeably exceeds its $G_{0} W_{0}$ values. The use of the local-field factor of Eq. (13) in $\Lambda^{\prime}$ results in the largest $Z_{F}$ among those presented in the table. Similar to the $G_{0} W \Lambda \& T$ case, the inclusion of the additional (magnetic) term shortens $\tau$ and reduces $Z_{F}$. However, if the reduction of the momentum distribution discontinuity is the same, the shortening of the lifetime is larger. As 
a cumulative result, we can infer that in the considered exciting-energy range the $G_{0} W \Lambda \& T$ and $G_{0} V_{\text {eff }}$ approaches are similar both by values (see Fig. 7) and physics behind them. At that the $G_{0} W \Lambda \& T$ approximation does not involve quantities, which are defined outside the scope of the approach.

\section{B. First-principles calculations}

In first-principles calculations, as a rule one starts not from the Hartree level, but from the local-density approximation (LDA). In this case the Kohn-Sham (KS) singleparticle wave functions $\psi_{\mathbf{k} n}^{K S}$ and energy eigenvalues $\epsilon_{\mathbf{k} n}^{K S}$ are regarded as good approximations to the quasiparticle ones. As a consequence, the many-body correction to $\epsilon_{\mathbf{k} n}^{K S}$ is defined by the matrix element $\left\langle\psi_{\mathbf{k} n}^{K S}|\Delta \Sigma(\omega)| \psi_{\mathbf{k} n}^{K S}\right\rangle$ of the difference $\Delta \Sigma=\Sigma-v_{\mathrm{LDA}}^{x c}$ between the self-energy and the XC potential $v_{\mathrm{LDA}}^{x c}$ obtained in the LDA. ${ }^{14}$ This leads to the inverse lifetime [Eq. (29)] given by

$$
\tau_{\mathbf{k} n}^{-1}=2 Z_{\mathbf{k} n}\left|\operatorname{Im} \Delta \Sigma_{\mathbf{k} n}\left(\epsilon_{\mathbf{k} n}^{K S}\right)\right|
$$

with the renormalization factor [Eq. (30)] rewritten as

$$
Z_{\mathbf{k} n}=\left[1-\frac{\partial \operatorname{Re} \Delta \Sigma_{\mathbf{k} n}(\omega)}{\partial \omega}\right]_{\omega=\epsilon_{\mathbf{k} n}^{K S}}^{-1} .
$$

The self-energy, in turn, is expressed in terms of the zerothorder Green's function $G_{0}$ built up from $\psi_{\mathbf{k} n}^{K S}$ and $\epsilon_{\mathbf{k} n}^{K S}$.

As follows from the above, the $G_{0} W_{0}$ and $G_{0} W_{0} \& T$ approximations set bounds to the results of the majority of the considered approaches. Therefore, in this work, we perform $a b$ initio calculations within these approximations only. We believe that it is well enough to accomplish our goal of comparison. Using the spectral function representation of $G_{0}$ and $W_{0}$, one can explicitly write down the imaginary part of the $G_{0} W_{0}$ self-energy as ${ }^{14}$

$$
\begin{aligned}
\operatorname{Im} \sum_{\mathbf{k} n}(\omega)= & \mp \sum_{\mathbf{q} n^{\prime}} \sum_{i j}\left\langle\psi_{\mathbf{k} n}^{K S} \psi_{\mathbf{q}-\mathbf{k} n^{\prime}}^{K S} \mid B_{\mathbf{q} i}\right\rangle \\
& \times \operatorname{Im}\left[W_{0}\right]_{i j}\left(\mathbf{q}, \pm \epsilon_{\mathbf{q}-\mathbf{k} n^{\prime}}^{K S} \mp \omega\right) \\
& \times\left\langle B_{\mathbf{q} j} \mid \psi_{\mathbf{q}-\mathbf{k} n^{\prime}}^{K S} \psi_{\mathbf{k} n}^{K S}\right\rangle \Theta\left( \pm \epsilon_{\mathbf{q}-\mathbf{k} n^{\prime}}^{K S} \mp \omega\right),
\end{aligned}
$$

where the upper (lower) sign corresponds to the exciting energy $\omega \leq E_{F}\left(\omega>E_{F}\right)$ and the sum over occupied (unoccupied) states. In Eq. (34), $\Theta(x)$ is the step function, $\left\{B_{\mathbf{k} i}(\mathbf{r})\right\}$ is a set of appropriate Bloch basis functions, and $\left[W_{0}\right]_{i j}$ are matrix elements of the RPA screened interaction in this basis. In the present work, the functions $\psi_{\mathbf{k} n}^{K S}$ and energies $\epsilon_{\mathbf{k} n}^{K S}$ are calculated by using both the linear muffin-tin orbital (LMTO) method and the plane-wave pseudopotential approach. Within the latter, the functions $B_{\mathbf{k} i}$ are plane waves with $i$ numbering reciprocal lattice vectors, whereas within the former they are represented by products of LMTO's. ${ }^{46}$ Having obtained the imaginary part of the self-energy, its energy-dependent real part can be found from the Hilbert transform.

In order to obtain the $T$-matrix contribution $\Sigma^{T}$, Eq. (20) is considered as a relation between the matrices $[T]_{i j}(\mathbf{q}, \omega)$,

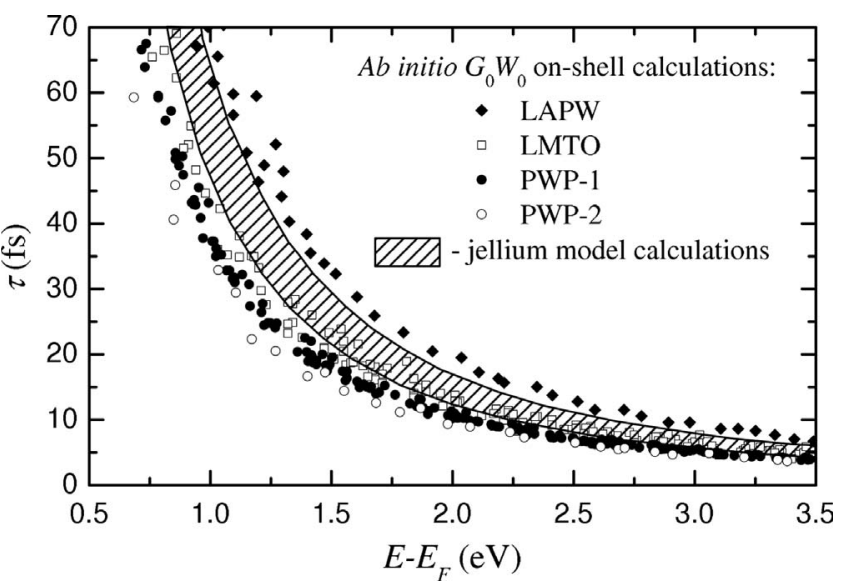

FIG. 8. The electron lifetime $\tau$ as a function of the exciting energy $E-E_{F}$ evaluated within the $G_{0} W_{0}$ on-shell approximation. The open squares represent the LMTO calculations. The solid circles depict the present calculation results obtained within the plane-wave pseudopotential approach (PWP-1). The open circles (labeled as PWP-2) correspond to results of Ref. 47. The solid diamonds represent the lifetime (some representative points) obtained in Ref. 48 with the use of the LAPW method. The shaded area bounded by two jellium-model curves " $G_{0} W_{0}$ " and " $G_{0} W_{0}$ on-shell" includes jellium-model calculations (see Fig. 7).

$[\widetilde{W}]_{i j}(\mathbf{q}, \omega)$, and $\left[P_{0}\right]_{i j}(\mathbf{q}, \omega)$ found in the basis $\left\{B_{\mathbf{k} i}(\mathbf{r})\right\}$. Further, Eq. (34) is used with $[T]_{i j}$ instead of $\left[W_{0}\right]_{i j}$. The main problem here is to calculate the local interaction $[\widetilde{W}]_{i j}$. Taking into account the results of Sec. II A 8, we model this interaction as $[\widetilde{W}]_{i j}=\frac{2}{3}\left[W_{0}\right]_{i j}(\mathbf{q}, 0)$. As a result, we have a possibility to include real band-structure effects into the factor $\mathcal{G}_{X}$.

Having obtained the self-energy, one can evaluate the lifetime using Eqs. (32) and (33). In order to compare the obtained results with the jellium-model curves, we average the calculated lifetime over momenta for a given exciting energy.

In Fig. 7, we show the LMTO results on momentumaveraged electron lifetimes in aluminum. Here we see that these $a b$ initio results reproduce rather well the jelliummodel curves. As in the jellium model, we also reveal that the on-shell $G_{0} W_{0}$ and $G_{0} W_{0} \& T$ results practically coincide. This means that within our numerical accuracy we do not find any considerable effect caused by taking the real band structure of aluminum into account.

However, it is worth emphasizing that the lifetime values evaluated within the same approximation (e.g., the on-shell $G_{0} W_{0}$ one) vary with the method for band-structure calculations. As an example, in Fig. 8, we compare the LMTO results with the plane-wave pseudopotential lifetimes (calculations of the present work are marked as PWP-1, while PWP-2 labels data taken from Ref. 47) and lifetimes obtained in Ref. 48 within the linearized augmented planewave method (LAPW). Other published ab initio results (e.g., Ref. 49) correspond to the data spread plotted. As is evident from the figure, one can draw different conclusions about the role of real band-structure effects. Actually, these effects can lead to both shortening the quasiparticle lifetime 
and making it longer. Nevertheless, as an analysis of the calculated scaled lifetime has shown, all the presented $a b$ initio data demonstrate the free-electron-like behavior of $\tau$ as a function of the exciting energy with underlying scattering processes peculiar to jellium.

\section{ELECTRON-PHONON SCATTERING}

The phonon-induced lifetime broadening can be evaluated from the imaginary part of the e-ph self-energy as ${ }^{50}$

$$
\begin{aligned}
\Gamma_{\mathrm{e}-\mathrm{ph}}(\epsilon)= & 2 \pi \int_{0}^{\omega_{m}} d \omega \alpha^{2} F(\epsilon ; \omega) \\
& \times[1+2 n(\omega)-f(\epsilon-\omega)+f(\epsilon+\omega)] .
\end{aligned}
$$

Here, $f$ and $n$ are the Fermi and Bose distribution functions, respectively, which introduce the temperature dependence of $\Gamma_{\text {e-ph }}$, and $\omega_{m}$ is the maximum phonon frequency. The basic quantity is the Eliashberg spectral function which measures the contribution of phonons with energy $\hbar \omega$ to the scattering of electrons:

$$
\begin{aligned}
\alpha^{2} F(\epsilon ; \omega)= & \frac{1}{N(\epsilon)} \sum_{\mathbf{q}, \nu} \delta\left(\omega-\omega_{\mathbf{q}, \nu}\right) \sum_{\mathbf{k}_{i}, \mathbf{k}_{f}}\left|g\left(\mathbf{k}_{i}, \mathbf{k}_{f}, \mathbf{q}, \nu\right)\right|^{2} \\
& \times \delta\left(\epsilon_{\mathbf{k}_{i}}-\epsilon\right) \delta\left(\epsilon_{\mathbf{k}_{f}}-\epsilon\right),
\end{aligned}
$$

where $g\left(\mathbf{k}_{i}, \mathbf{k}_{f}, \mathbf{q}, \nu\right)$ is the e-ph matrix element which indicates the probability of electron scattering from initial state $\mathbf{k}_{i}$ to final state $\mathbf{k}_{f}$ by phonon $(\mathbf{q}, \nu)$. The sum is carried out over initial and final electron states with energy $\epsilon$ to obtain the spectral function averaged over electron momentum. $N(\epsilon)$ is the electron density of states per atom and per spin at energy $\epsilon$. In Eq. (36) we apply the quasielastic approximation $\delta\left(\epsilon_{\mathbf{k}_{i(f)}}-\epsilon \pm \omega_{\mathbf{q}, \nu}\right) \approx \delta\left(\epsilon_{\mathbf{k}_{i(f)}}-\epsilon\right)$, which allows to calculate $\Gamma_{\text {e-ph }}$ with the same Eliashberg spectral function for both emission and absorption processes. The strength of the e-ph coupling $\lambda$ is defined as a first inverse frequency moment of the spectral function: ${ }^{50}$

$$
\lambda(\epsilon)=2 \int_{0}^{\omega_{m}} \frac{\alpha^{2} F(\epsilon ; \omega)}{\omega} d \omega .
$$

The calculations were performed by using the densityfunctional perturbation theory ${ }^{51,52}$ and the PWSCF code. ${ }^{53}$ The electron-ion interaction was described by a nonlocal normconserving pseudopotential generated following von Barth and Car. $^{53}$ To average the e-ph spectral function over all the initial states we used a dense mesh of up to $900 \mathbf{k}$ points in the irreducible Brillouin zone (IBZ). The summation over phonons $(\mathbf{q}, \nu)$ in Eq. (36) was carried out over 256 wave vectors in the irreducible part. Since the set of wave vectors sampled in the IBZ is large enough the results depend only slightly on the value of the smearing width.

We have calculated $\Gamma_{\text {e-ph }}(\epsilon)$ as a function averaged over electron momentum. Figure 9(a) shows the lifetime broadening of excited electrons at different temperatures. As one can see, $\Gamma_{\text {e-ph }}(\epsilon)$ varies slightly with energy outside the Debye energy. In particular, the variation range does not exceed 10 $\mathrm{meV}$ at $T=0$ and comes up to $40 \mathrm{meV}$ at room temperature.
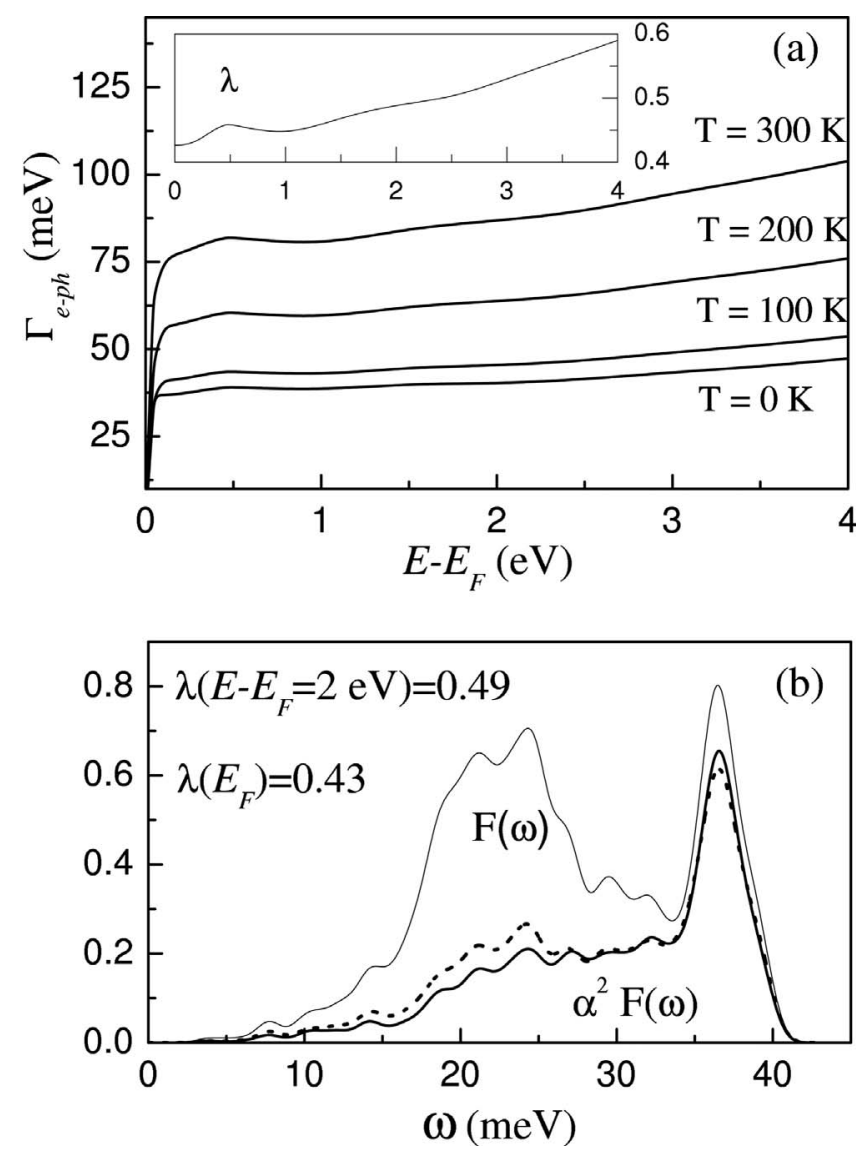

FIG. 9. (a) Phonon-induced lifetime broadening $\Gamma_{\text {e-ph }}$ averaged over electron momentum as a function of exciting energy at different temperatures. The inset gives the energy dependence of the corresponding e-ph coupling parameter $\lambda$. (b) Phonon density of states $F(\omega)$ (thin line) and e-ph spectral function $\alpha^{2} F(\epsilon ; \omega)$ at $E_{F}$ (solid line) and at $E-E_{F}=2 \mathrm{eV}$ (dashed line).

For exciting energies of $0.1-2.5 \mathrm{eV} \Gamma_{\mathrm{e}-\mathrm{ph}}(\epsilon) \approx 40 \mathrm{meV}$ at $T=0$ and then increases slowly with $\epsilon$. The calculated e-ph coupling parameter $\lambda(\epsilon)$ averaged over momentum for the same energies varies between 0.44 and 0.59 [see the inset of Fig. 9(a)]. The values of $\lambda$ at $0.1-2.5 \mathrm{eV}$ nearly coincide with the e-ph coupling parameter evaluated at the Fermi level of $\mathrm{Al}, \lambda\left(E_{F}\right)=0.43$. The temperature dependence of the e-ph contribution is evident. At room temperature, $\Gamma_{\mathrm{e}-\mathrm{ph}}(\epsilon)$ ranging from $66 \mathrm{meV}$ to $104 \mathrm{meV}$ becomes twice as much as the e-ph contribution at $T=0$.

The obtained data can also be momentum resolved to show the dependence of the e-ph coupling on the energy band. For example, Fig. 10 shows the lifetime broadening for excited electronic states in the electron bands $Z_{3}, Z_{4}$, and $Z_{1}$ (XW symmetry direction) of $\mathrm{Al}$ at $T=0 \mathrm{~K}$. As evident from the figure, the dependence of the e-ph contribution $\Gamma_{\mathrm{e}-\mathrm{ph}}(\epsilon)$ on the energy band $\left(Z_{4}\right.$ or $\left.Z_{1}\right)$ is rather appreciable. The values at the same energy can vary, for example, by $24 \mathrm{meV}$ at $\epsilon=1.3 \mathrm{eV}$ and by $5 \mathrm{meV}$ at $4 \mathrm{eV}$.

Figure 9(b) presents the momentum averaged e-ph spectral function for $\epsilon=2 \mathrm{eV}$ which turns out to be very similar to $\alpha^{2} F(\epsilon ; \omega)$ at $E_{F}$ also shown in the figure. The difference is noticeable only at low energies where the contribution of the 


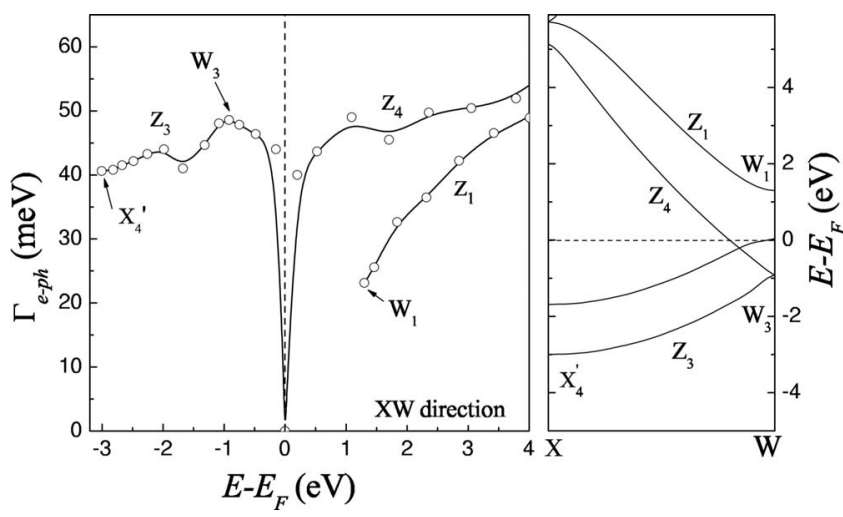

FIG. 10. Phonon-induced lifetime broadening $\Gamma_{\mathrm{e}-\mathrm{ph}}$ as a function of exciting energy for electronic energy bands $Z_{3}, Z_{4}$, and $Z_{1}$ (XW symmetry direction of the Brillouin zone) in $\mathrm{Al}$ at $T=0 \mathrm{~K}$.

corresponding phonon modes increases gradually with electronic state energy. On the whole, the high-energy phonons are more involved in the scattering processes of electrons than the low-energy part of the phonon spectrum which is strongly suppressed by e-ph matrix elements not only on the Fermi surface. The same feature was also reported for other simple metals Be (Ref. 54) and Mg (Ref. 55). It is not the case for $d$-metals like $\mathrm{Pd}$, where the phonon density of states and the Eliashberg spectral function at $E_{F}$ are very similar in shape though the high-energy part of phonons is suppressed more by e-ph matrix elements than the low-energy modes. ${ }^{8}$

\section{DISCUSSION AND CONCLUSIONS}

In this work, we have considered theoretical approaches to the quasiparticle lifetime (decay rate), which are most frequently used in the literature. We have also proposed an approach based on a variational solution to the Bethe-Salpeter equation defining the electron-hole scattering amplitude in the ladder approximation. With the screened Coulomb interaction evaluated beyond the RPA, the quasiparticle selfenergy of the approach consists of the $G_{0} W \Lambda$ part, which accounts for the leading contribution of charge-density fluctuations, and the $T$-matrix part, which enables one to take into consideration the contribution of spin-density fluctuations. Within the jellium model, we have demonstrated that, at exciting energies less than $3.5 \mathrm{eV}$, all the considered approaches yield rather close results on the quasiparticle lifetime in aluminum. An exception is the method that sums ladder self-energy diagrams by using the half inverse static dielectric function as an exchange part of the many-body local-field factor.

We have shown that the on-shell $G_{0} W_{0}$ approximation that ensures the leading contribution to many-body corrections simulates additional decay channels which are not implied by the $G_{0} W_{0}$ diagram. Regarding the question of how different expressions for the local-field factors influence the self-energy within the same approach, we have revealed that the approaches, which take into consideration the contribution of spin-density fluctuations, are the most sensible ones. Also, we have analyzed what effect the mentioned fluctua-

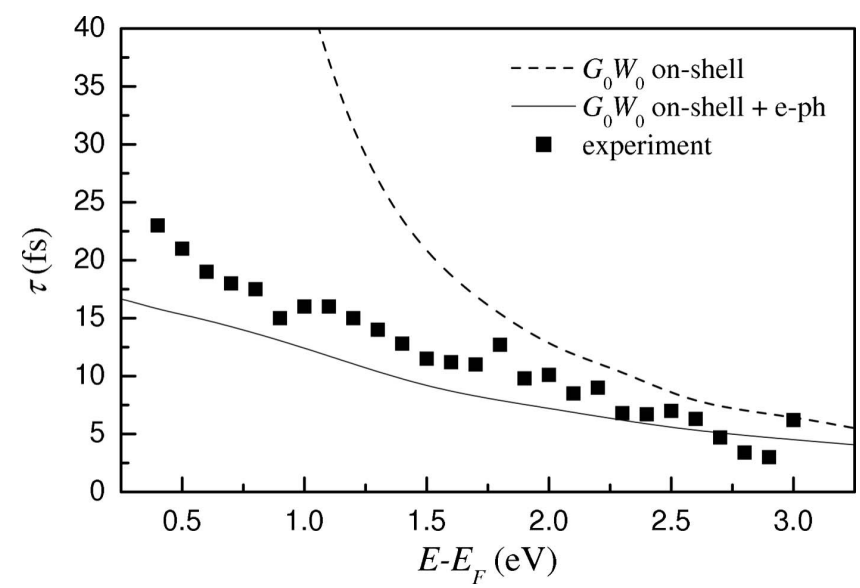

FIG. 11. The electron lifetime $\tau$ as a function of the exciting energy $E-E_{F}$. The dashed line represents the LMTO lifetime calculated within the on-shell $G_{0} W_{0}$ approximation. The solid line corresponds to the sum of the inelastic lifetime broadenings $\Gamma_{\mathrm{e}-\mathrm{e}}^{G_{0} W_{0}}$ and $\Gamma_{\text {e-ph }}($ at $T=0 \mathrm{~K})$. The solid squares are experimental data taken from Ref. 56

tions produce on quasiparticle properties. We find that the more scattering processes are involved in the electron-hole interaction, the greater the renormalization constant is. At the same time, the contribution to the imaginary part of the selfenergy has a trend to become smaller within the considered exciting-energy range.

We have performed $a b$ initio calculations of the lifetime in aluminum with the use of both the linear muffin-tin orbital method and the plane-wave pseudopotential theory. In the first case, the $a b$ initio results are similar to those obtained within the jellium model, whereas in the second case there is a decrease in lifetime values by $\sim 15 \%$. By this we demonstrate the dependence of real band-structure effects on practical schemes and computational details.

Also, the phonon contribution to the lifetime has been considered. On the basis of $a b$ initio calculations, we have shown that, except for the Fermi energy vicinity, the phononinduced lifetime broadening $\Gamma_{\mathrm{e}-\mathrm{ph}}(\epsilon)$ averaged over momentum varies slightly with exciting energy. However, the momentum resolved e-ph contribution as a function of exciting energy exhibits different behavior depending appreciably on electron energy band. The temperature dependence of $\Gamma_{\text {e-ph }}(\epsilon)$, which becomes twice as much at room temperature as compared with the e-ph contribution at $T=0 \mathrm{~K}$, is also evident. The analysis of phonon modes participating in the scattering processes of electrons has shown that the main contribution comes from the high-energy part of the phonon spectrum whereas the low-energy phonons are strongly suppressed by e-ph matrix elements at all the exciting energies considered.

In Fig. 11, we plot the ab initio results as compared with time-resolved two-photon photoemission (TR-2PPE) data available. ${ }^{56} \mathrm{As}$ is obtained for other systems, ${ }^{57} G_{0} W_{0}$ lifetimes (representing here the group of the considered approaches giving close lifetime values) are longer than experimental TR-2PPE relaxation times. In the case of aluminum, at $E-E_{F} \lesssim 1.7 \mathrm{eV}$, it becomes particularly evident. On the 


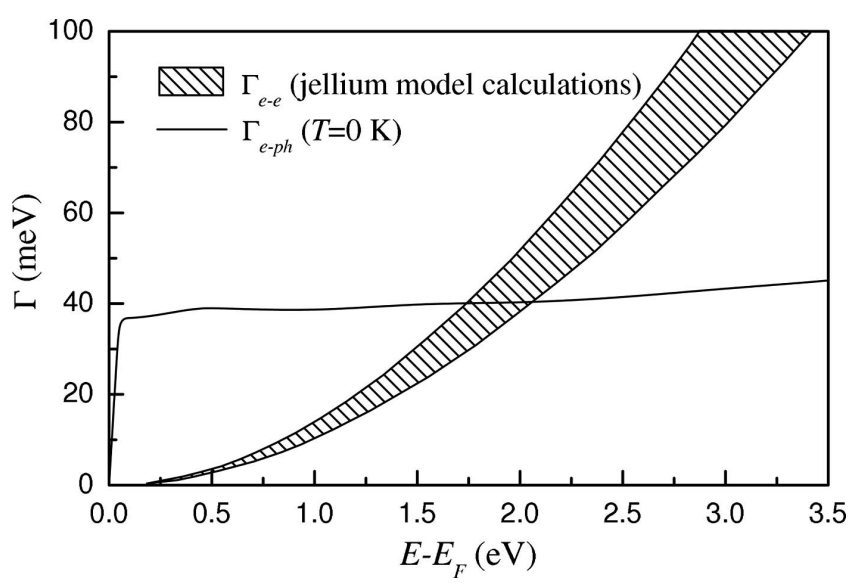

FIG. 12. The e-e (shaded area) and e-ph (solid line) contributions to the inelastic linewidth $\Gamma_{\text {tot }}$.

whole, the behavior of the TR-2PPE relaxation time is such that additional decay processes should be considered.

Taking into consideration both electron-electron and electron-phonon inelastic scattering processes, we arrive at electron lifetimes (solid line in Fig. 11) which demonstrate the energy dependence similar to that for the experimental relaxation times. Such a similarity is caused by a comparatively large e-ph contribution to the inelastic linewidth within the exciting-energy region, where the e-e contribution is rather small (see Fig. 12). As exciting energy increases, the linewidth due to the e-e scattering strengthens gradually, while $\Gamma_{\text {e-ph }}$ changes hardly. This results in considerable shortening of the quasiparticle lifetime at energies up to $E-E_{F}$ $\sim 2.0 \mathrm{eV}$ and in a fairly good agreement between the experimental data and theoretical results.
However, this agreement occurs for $\Gamma_{\mathrm{e}-\mathrm{ph}}$ at $T=0 \mathrm{~K}$, whereas at room temperature the calculated inelastic lifetime becomes notably shorter. This fact causes certain problems in definition of the proper approach by comparing to the experimental data. As to possible transport and cascade electron contributions to the relaxation time, a rough estimation does not help either to find the theoretical approach appropriate to the case.

In conclusion, the presented comparative analysis of different approximations of many-body perturbation theory as applied to aluminum has shown that the lifetime broadening coming from inelastic electron-electron scattering varies within $\sim 25 \%$ with the inclusion of scattering processes additional to those described within the $G_{0} W_{0}$ approximation. In comparison with the jellium model, taking the real band structure into account does not lead to any essential changes in the behavior of the lifetime as a function of exciting energy, except for some shortening. The behavior is notably changed by including the electron-phonon contribution: The total inelastic lifetime demonstrates relatively weak energy dependence and, with the phonon-induced broadening evaluated at $T=0 \mathrm{~K}$, decreases from $\sim 15$ fs to $\sim 5$ fs with exciting energy that increases from $0.5 \mathrm{eV}$ to $3.0 \mathrm{eV}$.

\section{ACKNOWLEDGMENTS}

We acknowledge partial support from the University of the Basque Country (Grant No. GIC07IT36607), the Basque Unibertsitate eta Ikerketa Saila, and the Spanish Ministerio de Ciencia y Tecnología (Grant No. FIS 2004-06490-C0301). The work of V.M.S. was supported by the IKERBASQUE Foundation.
*Theoretical Physics Department, Kostroma State University, 156961 Kostroma, Russia.

${ }^{1}$ E. V. Chulkov, A. G. Borisov, J.-P. Gauyacq, D. Sànchez-Portal, V. M. Silkin, V. P. Zhukov, and P. M. Echenique, Chem. Rev. (Washington, D.C.) 106, 4160 (2006).

${ }^{2}$ G. Vignale and K. S. Singwi, Phys. Rev. B 32, 2156 (1985); T. K. Ng and K. S. Singwi, ibid. 34, 7738 (1986).

${ }^{3}$ N. E. Bickers and S. R. White, Phys. Rev. B 43, 8044 (1991); N. E. Bickers, in Theoretical Methods for Strongly Correlated Electrons, edited by D. Sénéchal, A.-M. Tremblay, and C. Bourbonnais (Springer, New York, 2004).

${ }^{4}$ S. Yarlagadda and G. F. Giuliani, Phys. Rev. B 49, 14188 (1994).

${ }^{5}$ G. F. Giuliani and G. Vignale, Quantum Theory of the Electron Liquid (Cambridge University Press, Cambridge, England, 2005).

${ }^{6}$ B. Hellsing, A. Eiguren, and E. V. Chulkov, J. Phys.: Condens. Matter 14, 5959 (2002).

${ }^{7}$ E. V. Chulkov, J. Kliewer, R. Berndt, V. M. Silkin, B. Hellsing, S. Crampin, and P. M. Echenique, Phys. Rev. B 68, 195422 (2003).

${ }^{8}$ I. Yu. Sklyadneva, A. Leonardo, P. M. Echenique, S. V. Eremeev, and E. V. Chulkov, J. Phys.: Condens. Matter 18, 7923 (2006).
${ }^{9}$ Y. Baer and G. Busch, Phys. Rev. Lett. 30, 280 (1973).

${ }^{10}$ J. Sprösser-Prou, A. vom Felde, and J. Fink, Phys. Rev. B 40, 5799 (1989).

${ }^{11}$ K.-H. Lee and K. J. Chang, Phys. Rev. B 49, 2362 (1994).

${ }^{12}$ M. Quijada, A. G. Borisov, I. Nagy, R. Díez Muiño, and P. M. Echenique, Phys. Rev. A 75, 042902 (2007).

${ }^{13}$ L. Hedin, Phys. Rev. 139, A796 (1965).

${ }^{14}$ F. Aryasetiawan, in Strong Coulomb Correlations in Electronic Structure Calculations, edited by V. I. Anisimov (Gordon and Beach, Singapore, 2001).

${ }^{15}$ C. A. Kukkonen and A. W. Overhauser, Phys. Rev. B 20, 550 (1979).

${ }^{16}$ N. Iwamoto and D. Pines, Phys. Rev. B 29, 3924 (1984).

${ }^{17}$ M. Corradini, R. Del Sole, G. Onida, and M. Palummo, Phys. Rev. B 57, 14569 (1998).

${ }^{18}$ D. M. Ceperley and B. J. Alder, Phys. Rev. Lett. 45, 566 (1980); as parametrized by J. P. Perdew and A. Zunger, Phys. Rev. B 23, 5048 (1981).

${ }^{19}$ G. E. Simion and G. F. Giuliani, Phys. Rev. B 77, 035131 (2008).

${ }^{20}$ P. Gori-Giorgi and J. P. Perdew, Phys. Rev. B 64, 155102 (2001).

${ }^{21}$ I. A. Nechaev and E. V. Chulkov, Phys. Rev. B 73, 165112 
(2006).

${ }^{22}$ V. P. Zhukov, E. V. Chulkov, and P. M. Echenique, Phys. Rev. Lett. 93, 096401 (2004); Phys. Rev. B 72, 155109 (2005).

${ }^{23}$ In taking the Fourier transform, the vertex function $\Lambda(1,2,3)$ has been regarded as a function of 1-2 and 2-3.

${ }^{24}$ J. Hubbard, Proc. R. Soc. London, Ser. A 243, 336 (1957).

${ }^{25}$ G. D. Mahan, Comments Condens. Matter Phys. 16, 333 (1994).

${ }^{26}$ C. F. Richardson and N. W. Ashcroft, Phys. Rev. B 50, 8170 (1994).

${ }^{27}$ G. D. Mahan and B. E. Sernelius, Phys. Rev. Lett. 62, 2718 (1989).

${ }^{28}$ R. Del Sole, L. Reining, and R. W. Godby, Phys. Rev. B 49, 8024 (1994).

${ }^{29}$ M. Hindgren and C.-O. Almbladh, Phys. Rev. B 56, 12832 (1997).

${ }^{30}$ S. Doniach and S. Engelsberg, Phys. Rev. Lett. 17, 750 (1966).

${ }^{31}$ W. F. Brinkman and S. Engelsberg, Phys. Rev. 169, 417 (1968).

${ }^{32}$ J. A. Hertz and D. M. Edwards, Phys. Rev. Lett. 28, 1334 (1972).

${ }^{33}$ P. S. Riseborough, Phys. Rev. B 27, 5775 (1983).

${ }^{34}$ I. A. Nechaev and E. V. Chulkov, Phys. Rev. B 71, 115104 (2005).

${ }^{35}$ F. Bruneval, F. Sottile, V. Olevano, R. Del Sole, and L. Reining, Phys. Rev. Lett. 94, 186402 (2005).

${ }^{36}$ K. Karlsson and F. Aryasetiawan, Phys. Rev. B 62, 3006 (2000).

${ }^{37}$ F. Aryasetiawan, M. Imada, A. Georges, G. Kotliar, S. Biermann, and A. I. Lichtenstein, Phys. Rev. B 70, 195104 (2004).

${ }^{38}$ I. A. Nechaev, V. M. Silkin, and E. V. Chulkov, Phys. Solid State 49, 1820 (2007).

${ }^{39}$ G. D. Mahan, Many-Particle Physics (Plenum, New York, 1990).

${ }^{40}$ H. O. Frota and G. D. Mahan, Phys. Rev. B 45, 6243 (1992).

${ }^{41}$ A. Mönnich, J. Lange, M. Bauer, M. Aeschlimann, I. A.
Nechaev, V. P. Zhukov, P. M. Echenique, and E. V. Chulkov, Phys. Rev. B 74, 035102 (2006).

${ }^{42}$ J. J. Quinn and R. A. Ferrell, Phys. Rev. 112, 812 (1958).

${ }^{43}$ P. Gori-Giorgi and P. Ziesche, Phys. Rev. B 66, 235116 (2002).

${ }^{44}$ G. Ortiz and P. Ballone, Phys. Rev. B 50, 1391 (1994); 56, 9970 (1997).

${ }^{45}$ I. G. Gurtubay, J. M. Pitarke, and P. M. Echenique, Phys. Rev. B 69, 245106 (2004).

${ }^{46}$ F. Aryasetiawan and O. Gunnarsson, Phys. Rev. B 49, 16214 (1994).

${ }^{47}$ I. Campillo, V. M. Silkin, J. M. Pitarke, E. V. Chulkov, A. Rubio, and P. M. Echenique, Phys. Rev. B 61, 13484 (2000).

${ }^{48}$ F. Ladstädter, U. Hohenester, P. Puschnig, and C. AmbroschDraxl, Phys. Rev. B 70, 235125 (2004).

${ }^{49}$ W.-D. Schöne, R. Keyling, M. Bandić, and W. Ekardt, Phys. Rev. B 60, 8616 (1999).

${ }^{50} \mathrm{G}$. Grimvall, The Electron-Phonon Interaction in Metals (NorthHolland, Amsterdam, 1981).

${ }^{51}$ N. E. Zein, Fiz. Tverd. Tela (Leningrad) 26, 3028 (1984) [Sov. Phys. Solid State 26, 1825 (1984)].

${ }^{52}$ S. Baroni, S. de Gironcoli, A. Dal Corso, and P. Giannozzi, Rev. Mod. Phys. 73, 515 (2001).

${ }^{53}$ S. Baroni, S. de Gironcoli, A. Dal Corso, and P. Giannozzi (http://www.pwscf.org).

${ }^{54}$ I. Yu. Sklyadneva, E. V. Chulkov, W.-D. Schöne, V. M. Silkin, R. Keyling, and P. M. Echenique, Phys. Rev. B 71, 174302 (2005).

${ }^{55}$ A. Leonardo, I. Yu. Sklyadneva, V. M. Silkin, P. M. Echenique, and E. V. Chulkov, Phys. Rev. B 76, 035404 (2007).

${ }^{56}$ M. Bauer, S. Pawlik, and M. Aeschlimann, Proc. SPIE 3272, 201 (1998).

${ }^{57}$ See, e.g., I. A. Nechaev, E. V. Chulkov, and P. M. Echenique, Phys. Rev. B 76, 245125 (2007), and references therein. 